\title{
Actitudes de la ciudadanía hacia los servicios pú- blicos. Valoración y satisfacción en el periodo 2009-2011
}

\author{
Vanesa RODRÍGUEZ \\ Universidad de Oviedo \\ rodriguezvanesa@uniovi.es \\ Gabriel PRUNEDA \\ Universidad de Oviedo \\ pruneda@uniovi.es \\ Begoña CUETo \\ Universidad de Oviedo \\ bcueto@uniovi.es
}

Recibido: 29-11-2013

Aceptado: 02-06-2014

\section{Resumen:}

El objetivo con el que se plantea este artículo es estudiar cómo ha cambiado la satisfacción de los ciudadanos con el funcionamiento de los servicios públicos en España en el periodo 2009-2011, utilizando las Encuestas sobre "Calidad de los Servicios Públicos" que elabora el CIS en colaboración con la AEVAL. Los resultados muestran que ha aumentado ligeramente, a pesar del empeoramiento de la coyuntura económica. El nivel más elevado de satisfacción se registra para sanidad y educación, mientras que es menor en el caso de las prestaciones por desempleo y las pensiones. Los principales determinantes de una satisfacción alta con los servicios públicos son la ideología auto-declarada y la coyuntura económica. Por otra parte, los inmigrantes están más satisfechos que los españoles y el territorio tiene un efecto importante sobre la satisfacción.

Palabras clave: servicios públicos, satisfacción, crisis, orientación política 


\title{
Citizens' attitudes toward public services. Satisfaction in 2009-2011
}

\begin{abstract}
The aim of this paper is to study the recent changes on citizens' satisfaction with the performance of public services, in the period 2009-2011 in Spain. Using data from the surveys on Quality of Public Services, developed by the Sociological Research Center jointly with the National Agency for the Evaluation of Public Services and Quality of Services, our results show that the level of satisfaction has slightly increased, which seems to display a greater tendency to positively value public services during economic retrenchment. A major determinant of high satisfaction is self-reported ideology. Besides, immigrants display higher levels of satisfaction than Spaniards.
\end{abstract}

Keywords: citizen services, satisfaction, crisis, political orientation

\section{Referencia normalizada}

Rodríguez, V. et.al. (2014). "Actitudes de la ciudadanía hacia los servicios públicos. Valoración y satisfacción en el periodo 2009-2011”. Política y Sociedad, Vol 51, Núm. 2: 595-618

Sumario: Introducción. 1.Los estudios de opinión como fuente para entender las actitudes de la ciudadanía hacia los servicios públicos. 2.Fuentes, hipótesis y principales resultados. 3.Determinantes de la satisfacción con los servicios públicos. 4.Discusión y conclusiones. Bibliografía.

\section{Introducción}

En un contexto de severa crisis económica como el actual, la prestación de servicios públicos se encuentra en una encrucijada. Por una parte, los ingresos públicos se han reducido de forma sustancial, mientras que el gasto ha aumentado llevando a un incremento del déficit público que la Unión Europea exige sea reducido. Por otra, es en momentos de recesión como el que vivimos cuando las necesidades de cobertura social son demandadas de forma más intensa por los ciudadanos. En este marco, las políticas que los gobiernos están realizando suponen una reducción de dicho gasto, lo que afecta necesariamente a los servicios provistos por el Estado.

Tanto la reducción del gasto público como las reformas realizadas en los últimos años tienen efectos sobre el funcionamiento de los servicios públicos $\mathrm{y}$, por tanto, sobre la satisfacción con los mismos de la ciudadanía. Así, las encuestas de opinión que realiza anualmente el Centro de Investigaciones Sociológicas (CIS) constituyen un buen instrumento para estudiar las modificaciones que se han podido producir como consecuencia de los cambios habidos en el último quinquenio.

Del Pino y Díaz-Pulido (2011) señalan que el estudio de las encuestas de opinión debe tener en cuenta la comparación temporal para analizar cómo varían en el tiempo las actitudes de los ciudadanos. En este sentido, el objetivo general con el 
que se plantea este artículo es estudiar cuáles son las variables que influyen sobre la satisfacción con los servicios públicos. Y, un segundo objetivo es analizar cómo el actual contexto de crisis puede haber influido sobre la percepción de los servicios públicos por parte de la ciudadanía en términos de satisfacción con los mismos. Aunque la metodología utilizada en las encuestas del CIS no nos permite elaborar una serie longitudinal, puesto que no constituyen un panel de datos, sí se han mantenido preguntas homogéneas en el periodo 2009-2011, de forma que podemos analizar la evolución de la satisfacción ciudadana con el funcionamiento de los servicios públicos en este periodo de tiempo.

En particular, si los determinantes de la satisfacción son los mismos en los años considerados y si su efecto se mantiene en el tiempo o, por el contrario, cambia, aumentando la influencia de unas frente a otras.

En el siguiente apartado se sintetizan las principales conclusiones obtenidas por diversos autores sobre la valoración de los servicios públicos por parte de la ciudadanía. A continuación, se explican los datos utilizados y se muestra cómo ha cambiado la satisfacción con el funcionamiento de los servicios públicos en el periodo 2009-2011, para en el apartado que sigue mostrar los resultados del análisis estadístico realizado. Se finaliza con un breve apartado de conclusiones.

\section{Los estudios de opinión como fuente para entender las actitudes de la ciuda- danía hacia los servicios públicos}

Según Carrillo y Tamayo (2008), los estudios de opinión contribuyen, en mayor o menor medida, a revelar y comprender las preferencias de los ciudadanos respecto a los servicios públicos. Hay que tener en cuenta que la valoración de los distintos servicios públicos puede servir de apoyo a cambios en las políticas que realicen los gobiernos o, por el contrario, utilizarse como justificación para no realizar modificaciones o reformas aun cuando éstas resulten convenientes.

Varios autores han puesto de manifiesto que el temor de los políticos a ser castigados en las urnas provocaba que las reformas del Estado de Bienestar sean más bien limitadas. Las preferencias por el incremento del gasto público o, por el contrario, por reducciones en los impuestos, sin duda, influyen sobre las propuestas de reforma que puedan realizar los gobiernos. Así, el cambio en las actitudes de la ciudadanía, dejando de preferir el incremento del gasto social, podría aumentar las posibilidades de reformas sustanciales (Taylor-Gooby, 2001; del Pino, 2007; del Pino y Ramos, 2009).

La situación de crisis o de expansión económica también condiciona tanto la opinión de la ciudadanía como las propuestas de reforma por parte de los gobiernos. Del Pino et al. (2012) concluyen que el temor a que las reformas pasen factura en las próximas elecciones depende del ciclo económico. En periodos de crecimiento, la oposición de los votantes a las reformas del Estado de Bienestar convierten a éstas en un riesgo innecesario desde el punto de vista de los resultados electorales (incluso en gobiernos con mayoría parlamentaria). Sin embargo, en situaciones de 
crisis los gobiernos asumen riesgos electorales y deciden aplicar reformas tratando de elaborar un discurso político que reduzca el castigo electoral, puesto que el hecho de no hacer nada podría tener aún mayores consecuencias electorales.

Según del Pino (2007), las prestaciones y servicios públicos del Estado de Bienestar son consideradas por los ciudadanos como un derecho adquirido, no como una concesión caritativa por parte del Estado, lo que puede suponer un mayor rechazo a reformas que supongan una reducción de los servicios prestados. Fundación BBVA (2013) encuentra que, en España, existe una mayor preferencia por un sistema más amplio de Seguridad Social aunque ello suponga un aumento de la presión fiscal, frente a la opción de menores impuestos a costa de una limitación del sistema de Seguridad Social (en este caso, los resultados obtenidos en España son similares a los observados en los países nórdicos). Gran parte de los españoles considera que el Estado debe tener mucha responsabilidad, tanto en servicios vinculados al Estado de Bienestar, como en otros (esta demanda de intervención del Estado es claramente superior a la media europea). Así, la mayoría de los españoles se inclina hacia aumentar el gasto público en sanidad, atención a las personas con discapacidad y mayores, ayuda a los parados, educación e investigación científica. De la misma forma, más de la mitad de los encuestados consideran que se debería mantener el gasto en infraestructuras y medio ambiente, siendo deportes, junto con defensa, las partidas en las que una mayor parte de los ciudadanos considera que hay que reducir el gasto ( 31,9 y $40,3 \%$, respectivamente).

Aunque la mayor parte de la ciudadanía prefiera mantener o aumentar el gasto en servicios públicos, el apoyo es distinto según el tipo de servicios y también existen diferencias que vienen explicadas por diversos factores. A esta contención del gasto se puede llegar por dos vías, la reducción de los gastos (ya sea por reducción de las prestaciones o el incremento de los requisitos para acceder a ellas) o el incremento de los ingresos. Por ejemplo, según Fernández y Jaime-Castillo (2012) los ciudadanos son más propensos a apoyar un aplazamiento de la edad de jubilación en los países que ya presentan valores elevados; lo contrario ocurre con las cotizaciones sociales, puesto que en los países donde son más elevadas los ciudadanos son más reacios a apoyar nuevos incrementos. Sin embargo, en los países con una mayor pobreza, los ciudadanos son menos propensos a apoyar recortes en las prestaciones de jubilación.

Otro aspecto relevante en el análisis es la ideología. Blekesaune y Quadagno (2003) la tienen en cuenta a la hora de analizar las actitudes hacia las políticas de bienestar en 24 países. Estos autores no encuentran una relación directa entre las actitudes positivas hacia la sanidad y las pensiones y la ideología, (en este sentido, Jordan, 2010, encuentra una actitud más positiva hacia el sistema sanitario en 
aquellos países en los que la asistencia sanitaria está en manos del Estado $\left.{ }^{1}\right)$. Sin embargo, en las actitudes hacia la protección al desempleo observan una mayor variabilidad relacionada con la ideología, en situaciones de altos niveles de desempleo es mayor el apoyo a las políticas de bienestar dirigidas a los desempleados. En relación con el desempleo Fraile y Ferrer (2005), a partir del análisis de 13 países de la OCDE, encuentran resultados similares: los ciudadanos de países con altos niveles de desempleo son menos propensos a recortar el gasto en prestaciones por desempleo, mientras que en los países con bajos niveles de desempleo los ciudadanos son más proclives a este recorte.

González y Carreras (2012) indican que la ideología podría influir en la evaluación ciudadana por dos vías: sesgando el juicio de calidad, de forma que los ciudadanos con una posición ideológica más cercana al gobierno juzguen con mayor benevolencia la política desarrollada (hipótesis rechazada por la literatura al menos en el ámbito de los servicios locales). Por otro lado, la ideología, independientemente de que sesgue o no la percepción de la calidad de los servicios, puede atenuar o amplificar el impacto de la percepción sobre la decisión de voto. Estos autores estudian el efecto de la ideología en la calidad percibida en la administración local, encontrando que las actitudes hacia el gobierno dependen más de la percepción de los ciudadanos sobre la calidad de las políticas que de su orientación ideológica.

Por otra parte, la satisfacción con los servicios públicos puede depender no sólo de su calidad, sino también de las expectativas que se tienen sobre dichos servicios, tal y como señalan Roch y Poister (2006). De esta manera, a igual calidad de dos servicios proporcionará mayor satisfacción el que más se aproxime a las expectativas de los ciudadanos. Esta idea es compartida por Carrillo y Tamayo (2008), quienes indican que la baja satisfacción de los ciudadanos puede ser debida tanto a la baja eficacia percibida como a las elevadas expectativas existentes.

Las expectativas pueden estar relacionadas con la percepción que la ciudadanía tenga de la administración pública. Así, del Pino y Díaz-Pulido (2011) enumeran tres estereotipos de ciudadanos en función de su percepción de administración pública: caja negra, burocracia sin rostro y presunción de ineficacia. La caja negra hace referencia a la idea extendida de que la administración es un conjunto abstracto de organizaciones más preocupadas por los procedimientos en sí que por el fin al que sirven. La burocracia sin rostro está relacionada con la percepción de que los empleados públicos, y por ende la administración, carecen de sensibilidad respecto a los problemas de los ciudadanos. La presunción de ineficacia es la que lleva a pensar que tanto la administración como sus trabajadores son más ineficaces que la empresa privada.

${ }^{1}$ Este autor indica que la distinción entre sistemas sanitarios proporcionados por el Estado y aquellos que se combinan con los seguros privados no es sinónimo de los tres Estados de Bienestar de Esping-Andersen 
Sin embargo, numerosos estudios muestran que los ciudadanos, independientemente de su nivel educativo o clase social, prefieren la provisión pública de los servicios. Del Pino (2005b) indica que estos estereotipos aparecen cuando se habla de la administración pública en abstracto, mientras que si se estudian con mayor detalle las actitudes de los ciudadanos se observan mejores resultados. En un estudio sobre la relación entre la burocracia y la democracia en cinco países europeos e Israel, Vigoda-Gadot et al. (2010) encuentran una relación entre la eficiencia, eficacia y habilidad de la burocracia y el grado en que los ciudadanos tienen una visión favorable de la democracia. Según estos autores, para que los ciudadanos tengan confianza en el gobierno deben expresar su satisfacción con los servicios públicos y percibir una imagen favorable de los organismos estatales. En este sentido, respecto a la valoración que los ciudadanos hacen de la democracia, Fundación BBVA (2013) encuentra que los españoles, junto con los italianos, son los más descontentos con el funcionamiento de la democracia en sus respectivos países, mientras que daneses y suecos son quienes presentan una satisfacción más elevada.

Otro aspecto que se ha estudiado es la relación entre la eficacia percibida de los servicios públicos y la percepción de recursos insuficientes dedicados a estos servicios. Del Pino (2005a) y del Pino y Ramos (2009) señalan el elevado apoyo de los ciudadanos a sectores de la política social como son sanidad, pensiones y protección por desempleo, así como la idea generalizada de insuficiencia de gasto en estas políticas. En general, el porcentaje de ciudadanos que consideran el gasto excesivo es muy limitado. Con datos de 2005, Calzada y del Pino (2008) encuentran que son los ciudadanos más insatisfechos con los servicios públicos los que en mayor medida consideran que se deberían incrementar los recursos públicos a ellos destinados. En dicho año, el $69,2 \%$ de los ciudadanos preferían pagar más impuestos a cambio de mejores servicios (Calzada, 2007). La ideología contribuye a explicar la preferencia por bajar impuestos, aunque suponga una reducción de la prestación de servicios, de forma que las personas que se auto-ubican a la derecha del eje ideológico prefieren esta opción, de acuerdo con del Pino (2007). La misma autora indica que los españoles no comparten la idea de que, para mejorar la calidad y cantidad de los servicios del Estado de Bienestar, sea necesario aumentar los impuestos, sino que se deben gestionar mejor los recursos existentes.

No obstante, en un informe publicado en 2009 por la Agencia Estatal de Evaluación de las Políticas Públicas y la Calidad de los Servicios (AEVAL), titulado "La percepción Social de los Servicios Públicos en España (1985-2008)", se obtenía que los ciudadanos que perciben una menor eficacia de los servicios públicos son más proclives a valorar como escasos los recursos públicos destinados a estos recursos.

En dicho informe, los datos mostraban que la ciudadanía estaba satisfecha con el funcionamiento de los servicios públicos, aunque en los últimos años se percibía un incremento del porcentaje de ciudadanos insatisfechos. En cuanto a la opinión sobre el gasto en servicios públicos, éste ha variado en los últimos años: entre 1985 y 1989 aumentó la proporción que opinaba que se gastaba demasiado poco, en la década de los 90 se observa la tendencia contraria y, a partir de 1999, vuelve a aumentar el porcentaje de ciudadanos que considera que el gasto es insuficiente. 
La opinión sobre la suficiencia o no del gasto y la disposición a pagar más o no, está relacionada con la preferencia por la provisión pública o privada de determinados servicios. En este sentido, los españoles prefieren fórmulas de gestión de los servicios principalmente públicas, a pesar de que consideran que el sector privado es más eficaz. Consideran que se puede compatibilizar con la gestión privada siempre que ésta tenga un peso bajo (del Pino, 2007). Esta preferencia por la gestión pública es mayor entre los ciudadanos que se auto-ubican en la parte baja de la escala social.

Finalmente, hay que tener en cuenta que algunos servicios públicos son provistos a nivel nacional mientras que en otros la competencia recae en las comunidades autónomas o en los municipios. Díaz-Pulido et al. (2012) encuentran que el grado de satisfacción de los ciudadanos difiere entre las distintas comunidades autónomas, principalmente en el caso de los servicios de competencia autonómica y que, además, estas diferencias no desaparecen al controlar por variables sociodemográficas, económicas o ideológicas.

En general, los resultados son diversos, pero podemos señalar algunos aspectos comunes. Por ejemplo, los ciudadanos que se autoubican a la derecha del eje ideológico presentan menores niveles de satisfacción con la mayor parte de los servicios públicos. Asimismo, las expectativas sobre la calidad de los mismos también afecta a su valoración, que puede cambiar en función de la coyuntura económica y el uso de los distintos servicios. En este sentido, la aportación de este artículo pasa por el análisis de la satisfacción con los servicios públicos en la sociedad española en un momento económico muy particular, dado el efecto que la crisis económica está teniendo sobre el Estado de Bienestar.

\section{Fuentes, hipótesis y principales resultados}

Tal y como se ha indicado en la introducción, el objetivo general de este artículo es analizar cómo el actual contexto de crisis económica ha influido sobre la percepción de los servicios públicos por parte de la ciudadanía, en términos de satisfacción con los mismos. Así, en este apartado se analiza la evolución de la satisfacción con la calidad de los servicios públicos en el periodo 2009-2011. Para ello, se utilizan las Encuestas sobre "Calidad de los Servicios Públicos" que elabora el CIS en colaboración con la AEVAL. Se trata de los estudios 2.813, 2.840 y 2.908. Aunque el cuestionario no es idéntico, sí se mantienen preguntas comunes que permitirán estudiar la evolución en el tiempo de ciertas variables relacionadas tanto con la satisfacción con los servicios públicos como con la valoración de los mismos por parte de la ciudadanía. Asimismo, se mantienen preguntas que permiten disponer de 
información sobre las características sociodemográficas de las personas encuestadas y sobre su ideología ${ }^{2}$.

La pregunta a partir de la cual es posible estudiar el grado de satisfacción de las personas con los servicios públicos es la siguiente: "Pensando ahora en los servicios públicos que prestan tanto el Estado como las Comunidades Autónomas y los Ayuntamientos y de los que hemos ido hablando en este cuestionario, por su propia experiencia o por lo que tiene entendido, ¿diría Ud. que, en general, los servicios públicos funcionan de forma muy satisfactoria, bastante, poco o nada satisfactoria?". En una escala de 1 a 4, donde el 1 significa que los servicios públicos funcionan de forma 'muy satisfactoria' y el 4 de forma 'nada satisfactoria', la media en 2009 y en 2010 se sitúa en 2,47, mientras que en 2011 mejora alcanzando un 2,36. Es decir, en el último año considerado, se constata un aumento del porcentaje de ciudadanos que responden que están 'muy satisfechos' o 'bastante satisfechos' con el funcionamiento de los servicios públicos.

Gráfico 1. Porcentaje de ciudadanos 'muy satisfechos' o 'bastante satisfechos' con el funcionamiento de los servicios públicos.

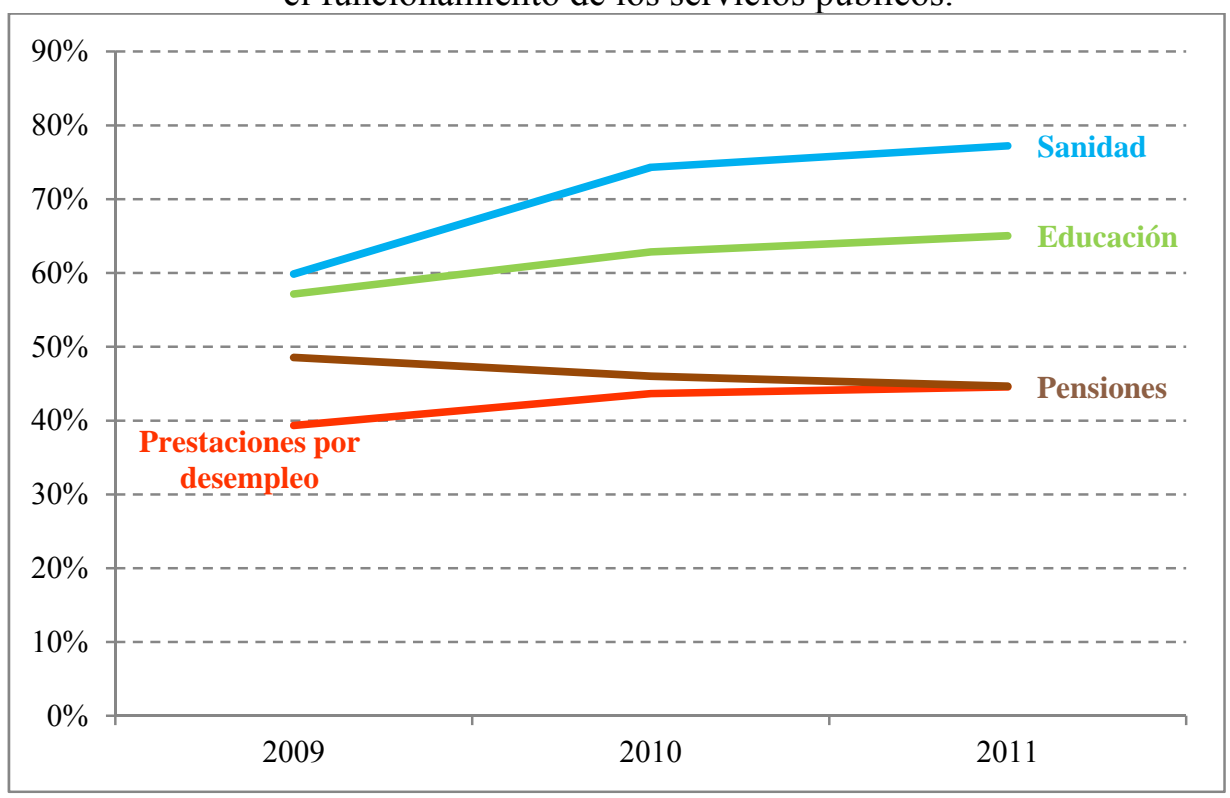

Fuente: Encuestas sobre "Calidad de los Servicios Públicos” 2009-2011. CIS.

${ }^{2}$ En el Anexo se incluye una tabla con los estadísticos descriptivos de las variables utilizadas (Tabla A.I). 
Además de esta pregunta general, se realiza otra sobre el funcionamiento de varios servicios públicos, cuyo enunciado es el siguiente: "Independientemente de que los utilice, ¿está Ud. muy satisfecho/a, bastante, poco o nada satisfecho/a con el funcionamiento de los siguientes servicios públicos?". El listado incluye diez servicios de los cuales hemos seleccionado cuatro: educación, sanidad, prestaciones por desempleo y pensiones ${ }^{3}$. Se trata de los servicios más directamente relacionados con el Estado de Bienestar y son los que suponen un mayor porcentaje del gasto público. En el Gráfico 1 se muestra el porcentaje de ciudadanos 'muy' o 'bastante' satisfechos con el funcionamiento de los mismos. Se puede observar que sanidad y educación presentan los niveles de satisfacción más elevados, con un considerable aumento en 2009 y estabilidad en 2010. En cambio, en el caso de las prestaciones por desempleo y las pensiones, el porcentaje de ciudadanos 'muy satisfechos' o 'bastante satisfechos' con el funcionamiento de estos servicios no llega al $50 \%$, habiéndose reducido ligeramente en el caso de las pensiones ${ }^{4}$.

La revisión de la literatura previa ha puesto de manifiesto la existencia de diferencias en la satisfacción con los servicios públicos en función de distintas variables. En el Gráfico 2 y la Tabla I se muestra el porcentaje de ciudadanos satisfechos con los distintos servicios públicos según su situación laboral. Así, el mayor porcentaje de ciudadanos 'muy satisfechos' o 'bastante satisfechos' con el funcionamiento de los servicios públicos corresponde a quienes se encuentran en situación de inactividad, ya sea por percibir una pensión o por otra razón. En el extremo opuesto se encuentran las personas en situación de desempleo, grupo en el que en torno a un 50\% manifiesta estar 'muy satisfecho' o 'bastante satisfecho' con el funcionamiento de los servicios públicos. En todos los casos, se observa una tendencia creciente, como la ya indicada para el conjunto de la ciudadanía.

En los cuatro servicios considerados, los pensionistas y otros inactivos son los colectivos que presentan un porcentaje más elevado de ciudadanos satisfechos. En cambio, ocurre lo contrario con las personas en situación de desempleo, que muestran porcentajes más bajos de ciudadanos 'muy' o 'bastante satisfechos' con el funcionamiento de los servicios públicos. En este colectivo, dicha proporción es

${ }^{3}$ El enunciado exacto de cada uno de los servicios públicos considerados es: "la enseñanza pública", "la sanidad pública", "la gestión de las ayudas de protección por desempleo" y "la gestión de las pensiones". Por tanto, en el caso de las prestaciones de la protección por desempleo y de las pensiones, se hace referencia a su gestión. No obstante, de cara al análisis de la satisfacción con los servicios públicos, creemos que la ciudadanía responde, en todos los casos, con una valoración general del servicio

${ }^{4}$ Las series del Banco de Datos del CIS permiten un análisis temporal más amplio. Sin embargo, no se cuenta con los microdatos necesarios para realizar el análisis posterior, por lo que este artículo se llevará a cabo para el periodo 2009-2011. En el caso de satisfacción con la enseñanza, servicio para el que existen datos correspondientes a 2012 se observa una reducción del porcentaje de ciudadanos "muy" o "bastante" satisfechos. 
especialmente baja en el caso de las prestaciones por desempleo, servicio en el que menos de un $40 \%$ declara estar 'muy' o 'bastante satisfecho' con el funcionamiento de dicho servicio.

Gráfico 2. Porcentaje de ciudadanos 'muy satisfechos' o 'bastante satisfechos' con el funcionamiento de los servicios públicos, según su situación laboral.

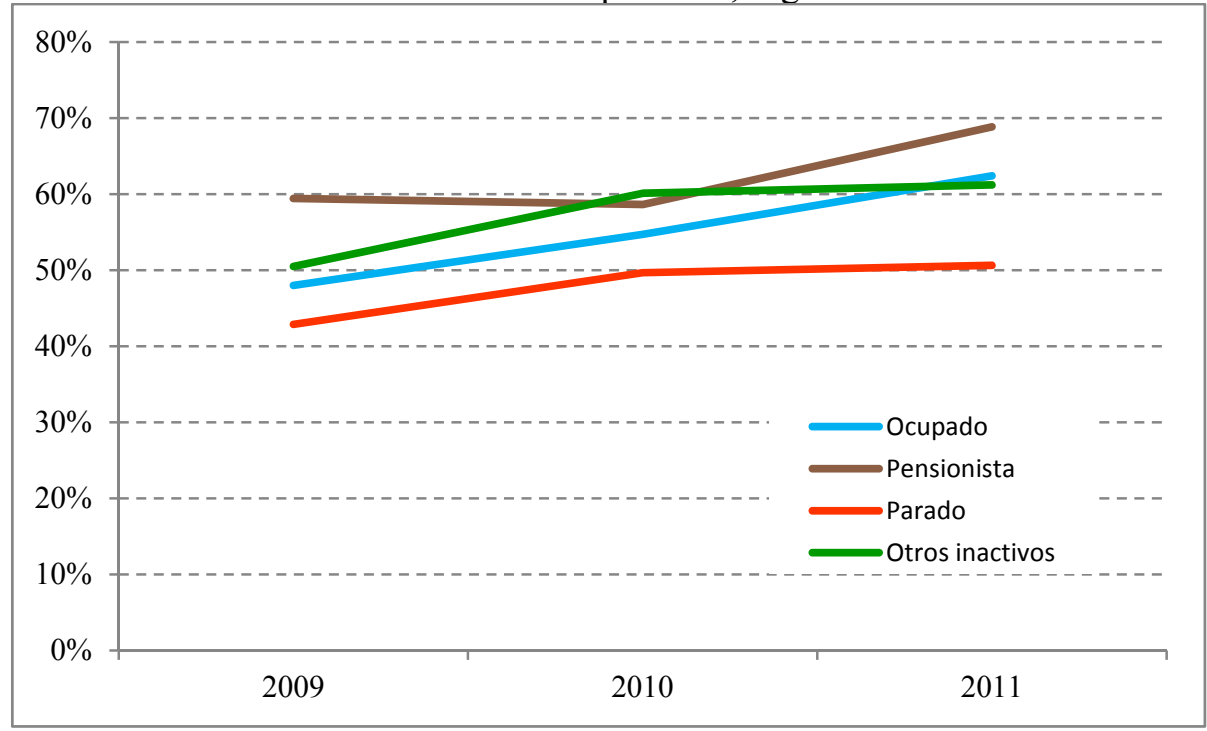

Fuente: Encuestas sobre "Calidad de los Servicios Públicos” 2009-2011. CIS.

Tabla I. Porcentaje de ciudadanos 'muy satisfechos' o 'bastante satisfechos' con el funcionamiento de distintos servicios públicos, según su situación laboral.

\begin{tabular}{|l|ccc|ccc|}
\hline & \multicolumn{5}{|c|}{ Educación } & \multicolumn{3}{c|}{ Sanidad } \\
Ocupado & $\mathbf{2 0 0 9}$ & $\mathbf{2 0 1 0}$ & $\mathbf{2 0 1 1}$ & $\mathbf{2 0 0 9}$ & $\mathbf{2 0 1 0}$ & $\mathbf{2 0 1 1}$ \\
Parado & 55,4 & 60,5 & 64,0 & 56,1 & 51,8 & 58,8 \\
Pensionista & 56,7 & 59,3 & 64,8 & 53,3 & 47,7 & 55,0 \\
Otros inactivos & 58,9 & 65,1 & 70,3 & 66,0 & 65,9 & 70,0 \\
& 59,7 & 73,8 & 61,9 & 60,1 & 57,7 & 61,2 \\
& \multicolumn{9}{|c}{ Prestaciones por desempleo } & \multicolumn{4}{c}{ Pensiones } \\
Ocupado & $\mathbf{2 0 0 9}$ & $\mathbf{2 0 1 0}$ & $\mathbf{2 0 1 1}$ & $\mathbf{2 0 0 9}$ & $\mathbf{2 0 1 0}$ & $\mathbf{2 0 1 1}$ \\
Parado & 40,5 & 46,7 & 43,5 & 48,5 & 45,7 & 43,2 \\
Pensionista & 36,1 & 39,0 & 39,0 & 48,3 & 42,3 & 37,1 \\
Otros inactivos & 44,5 & 44,2 & 50,2 & 50,7 & 47,0 & 48,7 \\
\hline
\end{tabular}

Fuente: Encuestas sobre "Calidad de los Servicios Públicos" 2009-2011. CIS. 
A partir de la revisión bibliográfica realizada en el apartado anterior y el análisis llevado a cabo hasta el momento, las hipótesis a contrastar en el apartado siguiente, utilizando el análisis estadístico, son las que siguen:

La distinta situación laboral de los ciudadanos puede dar lugar a distintas valoraciones de los servicios públicos, de forma que quienes contribuyen en mayor medida a los mismos pueden estar menos satisfechos como consecuencia de unas mayores expectativas. Así, la primera hipótesis se formula de la siguiente manera:

H1: la satisfacción con el funcionamiento de los servicios públicos es menor entre las personas ocupadas y desempleadas y mayor entre las inactivas.

Distintos autores han puesto de manifiesto que la ideología es uno de los factores que más influyen sobre la satisfacción con los servicios públicos. Por tanto, la segunda hipótesis es:

H2: la satisfacción con el funcionamiento de los servicios públicos es mayor entre

los ciudadanos que manifiestan una orientación ideológica "de izquierdas".

Dado el proceso de descentralización de determinados servicios públicos que ha tenido lugar en España, la valoración de los mismos puede ser diferente según la comunidad autónoma de residencia. Así, la tercera hipótesis se formula como sigue: H3: la influencia de la región de residencia sobre la satisfacción con los servicios públicos será mayor para los servicios que sean competencia de las comunidades autónomas.

Nuestra cuarta hipótesis se centra en la existencia de un colectivo importante de inmigrantes, proveniente en su mayoría de países con un Estado de Bienestar menos desarrollado que el existente en nuestro país:

H4: la satisfacción de las personas inmigrantes con los servicios públicos es más alta que la de los nacidos en España

Finalmente, teniendo en cuenta que la situación de crisis económica en el periodo 2009-2011 ha empeorado y que se han reducido los recursos dedicados a varios servicios públicos, es posible que la ciudadanía valore más positivamente la existencia en sí misma de dichos servicios. Por ello, la quinta hipótesis es la siguiente: H5: la satisfacción con el funcionamiento de los servicios relacionados con el Estado de Bienestar aumenta en momentos de crisis económica.

\section{Determinantes de la satisfacción con los servicios públicos}

En este apartado se lleva a cabo un análisis estadístico para estudiar los determinantes de la satisfacción con los servicios públicos considerados. Nuestra variable dependiente toma el valor 1 si la persona manifiesta estar "muy" o "bastante" satisfecha con los servicios públicos y valor $0 \mathrm{si}$, por el contrario, está "poco" o "nada" satisfecha. 
Dado que esta variable dependiente es categórica, utilizaremos modelos logit para estimar los efectos que distintas variables independientes tienen sobre la probabilidad de manifestar un grado de satisfacción alto. Entre las variables independientes se incluirán las características socioeconómicas de las personas (sexo, edad, nacionalidad, nivel de estudios, situación laboral, ocupación desarrollada y estatus), las relacionadas con el lugar de residencia (comunidad autónoma y tamaño del municipio), con la orientación ideológica de las personas y su participación en las últimas elecciones y, finalmente, con la coyuntura económica (tasa de paro regional y año de la encuesta).

En primer lugar, se ha realizado una estimación sobre la probabilidad de estar satisfecho con el funcionamiento de los servicios públicos en general y, en segundo lugar, con los cuatro servicios que hemos seleccionado: sanidad, educación, prestaciones por desempleo y pensiones. Como ya se ha indicado, estos son los cuatro servicios más directamente relacionados con el Estado de Bienestar y los que suponen un mayor porcentaje del gasto público. En la Tabla A.I.I del Anexo se muestran los resultados obtenidos.

Para facilitar la interpretación de los resultados, los coeficientes obtenidos se presentan en forma de ratios de probabilidad relativa (odds ratio). Esto implica que un coeficiente mayor de 1 representa una probabilidad superior a la de la categoría de referencia de estar satisfecho con el funcionamiento de los servicios públicos, mientras que si es menor de 1 ocurre lo contrario. A modo de ejemplo, tomaremos el ratio de probabilidad relativa asociado a la variable sexo en la regresión correspondiente a la satisfacción con los servicios públicos en general (véase Tabla A.II). La categoría de referencia es mujer, por lo que 1,082 significa que la probabilidad de una satisfacción alta es un $8,2 \%$ superior en el caso de los hombres.

En cuanto a la situación laboral, los desempleados muestran una probabilidad de estar altamente satisfechos un 32\% menor que la de los trabajadores en activo, mientras que para los inactivos (excluidos los pensionistas) es superior en un $14 \%$. Nuevamente, estos resultados van en la misma dirección que los obtenidos por del Pino (2007).

Este último colectivo es el que valora la sanidad pública de forma más positiva, mientras que los desempleados tienden a manifestarse menos satisfechos con el sistema de prestaciones por desempleo que quienes cuentan con trabajo.

En este caso, los resultados no nos permiten aceptar la hipótesis 1, puesto que la situación laboral resulta significativa únicamente en una de las categorías y sólo para uno de los servicios públicos estudiados.

Por otra parte, el hecho de haber votado en las últimas elecciones también contribuye a explicar el grado de satisfacción con los servicios públicos, teniendo quienes ejercieron su derecho al voto una probabilidad superior en un $17 \%$ de encontrarse altamente satisfechos frente a quienes no lo hicieron. La orientación política se sitúa como uno de los aspectos más relevantes a la hora de determinar la satisfacción con los servicios públicos, disminuyendo la probabilidad de que ésta sea considerablemente alta a medida que la ideología se hace más conservadora. En este sentido, y tomando como referencia a quienes claramente se manifiestan "de 
izquierdas", podemos observar que los individuos que se autodefinen "de derechas" tienen una menor probabilidad de expresar altos niveles de satisfacción con los servicios públicos en España. Este resultado podría deberse a que este colectivo suele corresponderse por definición con quienes abogan por una mayor privatización de "lo público", por tanto no valorando en exceso los servicios que el Estado presta a los ciudadanos. Este resultado coincide con los obtenidos por Díaz-Pulido et al. (2012), mientras que del Pino (2007) sólo encuentra esta característica relevante, y positiva, para explicar la satisfacción con el sistema de gestión de pensiones.

Sin embargo, la tendencia que observamos en el caso de aquéllos que se definen tanto de "centro" como de "centro-izquierda" es precisamente la contraria, ya que la probabilidad de que estén altamente satisfechos con los servicios públicos es superior a la de quienes se sitúan a la izquierda por completo. Detrás de este interesante resultado podría estar el hecho de que éstos son unos claros defensores del Estado de Bienestar en su máxima expresión.

En los resultados correspondientes a cada uno de los servicios públicos estudiados, se observa de manera clara entre la ciudadanía una tendencia a la baja de manifestarse altamente satisfecha con los cuatro servicios públicos considerados a medida que se sitúan a la derecha del eje ideológico. Estos resultados nos permiten aceptar la hipótesis 2 , relativa a la mayor satisfacción con los servicios públicos de los ciudadanos que se autodefinen "de izquierdas".

En cuanto a la comunidad autónoma de residencia, ésta resulta significativa en la mayoría de casos. Se observa que únicamente los ciudadanos de la Comunidad Valenciana, Extremadura y, en especial, Canarias, tienen una menor probabilidad de presentar niveles altos de satisfacción con los servicios públicos respecto a Madrid (categoría de referencia).

En las regresiones para cada uno de los servicios públicos considerados, tomando Madrid como referencia, tal y como se ha expuesto, únicamente los ciudadanos de Andalucía y, de manera especial los de Canarias, revelan una disminución en la probabilidad de disfrutar de altos niveles de satisfacción con el sistema sanitario (resultados coincidentes con los obtenidos por AEVAL, 2010). En lo que se refiere a la educación pública, todos los coeficientes son superiores a la unidad y estadísticamente significativos, con la salvedad del correspondiente a Canarias, que resulta no significativo. De esta manera, podemos afirmar que Madrid es la comunidad autónoma donde la probabilidad de que los ciudadanos estén altamente satisfechos con la educación es menor (de nuevo, un resultado en la línea de los obtenidos por Díaz-Pulido et al., 2012 y AEVAL, 2010).

En cuanto a las prestaciones por desempleo, obtenemos resultados significativos para ocho comunidades, todos ellos indicativos de una probabilidad superior a la de Madrid de tener niveles de satisfacción elevados. Concretamente, estas comunidades son Asturias, Aragón, Navarra, La Rioja, Castilla y León, Baleares, Galicia y Cataluña. Por último, las comunidades donde la valoración de las pensiones supera a la de Madrid son, por este orden, La Rioja, Asturias, Navarra, Castilla y León y Aragón. En general, la significatividad de los coeficientes correspondientes a las 
comunidades autónomas es mayor en sanidad y educación que en prestaciones por desempleo y pensiones. Esta mayor relevancia del territorio en los servicios trasferidos a las comunidades autónomas podría interpretarse relacionándola con la existencia de diferencias territoriales en la prestación de servicios. En cambio, en el caso de prestaciones por desempleo y pensiones, la provisión estatal de los mismos daría lugar a menos diferencias percibidas en la ciudadanía.

Estos resultados apoyarían la hipótesis 3, señalando la mayor importancia de la comunidad autónoma de residencia en los servicios públicos que están transferidos.

Por último, cabe destacar que el tamaño del municipio tiene un efecto negativo en la satisfacción con las prestaciones por desempleo y las pensiones. Esto puede deberse a la pérdida de poder adquisitivo que acarrea pasar de cobrar un salario a una pensión, pérdida que suele resultar más acusada en municipios de mayor tamaño, donde es habitual que el coste de vida sea más elevado.

El impacto de la nacionalidad sobre la satisfacción es muy notable, pues ser español reduce en casi cuatro veces la probabilidad de estar muy satisfecho. Estos resultados coinciden en gran medida con los obtenidos por del Pino (2007) y DíazPulido et al. (2012). Una posible explicación a esto sería el hecho de que gran parte de la población extranjera en España ha inmigrado a este país buscando una oportunidad de disfrutar de una calidad de vida superior a la que tenían en su país de origen.

Los resultados se repiten para cada uno de los servicios públicos estudiados. De hecho, los nacidos en España muestran en todos los casos una probabilidad de estar altamente satisfechos inferior a aproximadamente el doble de la de los extranjeros, acercándose al triple tanto en el caso de la educación pública como en el de las pensiones. Así, podemos aceptar la hipótesis 4, sobre la mayor satisfacción con los servicios públicos de las personas no nacidas en España.

La tasa de paro regional que se ha incluido en el modelo indica que el nivel de desempleo tiene valor explicativo de cara a la satisfacción de los individuos con los servicios públicos. Así, se observa que cuando el nivel de desempleo se encuentra entre el 15 y el $20 \%$, la probabilidad de que la satisfacción sea alta aumenta un $54 \%$ en comparación con niveles comprendidos entre el 10 y el $15 \%$, mientras que cuando es inferior al 10\% disminuye un 35\%. Es decir, la tasa de desempleo autonómica tiene capacidad predictiva a la hora de expresar altos niveles de satisfacción con la sanidad, las prestaciones por desempleo y las pensiones, de manera que cuanto más elevada es la tasa de paro, mayor es la probabilidad de una alta satisfacción. Esta variable no es significativa, sin embargo, en lo que al sistema educativo respecta.

De forma complementaria, la variable que recoge el año de la encuesta nos proporciona información en este mismo sentido, ya que la probabilidad de expresar altos niveles de satisfacción con los servicios públicos es inferior tanto en 2009 como en 2010 con respecto a 2011. Concretamente, estas disminuciones se cuantifican en un 59 y un $33 \%$, respectivamente.

En las regresiones para cada uno de los servicios públicos considerados, se observa una mejoría en la percepción existente acerca del sistema de sanidad pública, 
de la educación y de las prestaciones por desempleo en 2011 respecto a los años anteriores. Este resultado podría ser explicado por la amenaza de que estos servicios sean reducidos, que podría conducir a una mejor valoración de los mismos. En cambio, empeora la referida a las pensiones, probablemente debido a las últimas reformas en este sentido y quizás también a que los altos índices de desempleo repercuten sin lugar a dudas en las condiciones en que muchos ciudadanos accederán a este tipo de prestación.

Estos resultados apoyarían la hipótesis 5, que preveía un aumento de la satisfacción con los servicios públicos a medida que la crisis es más prolongada. Es decir, se observa un aumento en la apreciación de los servicios públicos por parte de los ciudadanos a medida que el empleo se destruye, lo que no debería extrañarnos dado que esta circunstancia convierte en usuarios de diversos tipos de prestaciones a quienes se ven afectados por este hecho, a la vez que es más fácil que valoren la calidad de la enseñanza o la sanidad públicas. Asimismo, podría estar contribuyendo a que quienes perciben una pensión sean más conscientes del elevado nivel de seguridad del que disfrutan o del escaso riesgo que corren. Estos resultados van en la línea de los obtenidos por Blekeasune y Quadagno (2003), que encuentran un mayor apoyo a las políticas de bienestar dirigidas a los desempleados en países de elevados niveles de desempleo. No obstante, el periodo analizado es corto y no se trata de datos longitudinales, por lo que estos resultados deben ser tomados con cautela.

Finalmente, algunos otros resultados que se desean destacar son que la probabilidad de expresar una alta satisfacción con los servicios públicos, en general, es ligeramente superior para los hombres y que, además, disminuye con la edad. Los hombres están más satisfechos que las mujeres con la sanidad, las prestaciones por desempleo y el sistema de pensiones, mientras que en el caso de la educación pública son las mujeres quienes están más satisfechas que los hombres. Una posible interpretación de este resultado consistiría en que la mejor situación de los hombres dentro del mercado de trabajo conlleva su acceso tanto a la prestación por desempleo como a las pensiones en condiciones más favorables que las de las mujeres, utilizando además el sistema de sanidad en menor medida que éstas. Por su parte, las mujeres mostrarían una mayor satisfacción con la educación pública por ser usuarias en mayor medida que los hombres. La edad influye negativamente en el nivel de satisfacción, excepto en el caso de las prestaciones por desempleo, donde no existen diferencias significativas (resultado que no coincide con los obtenidos por del Pino, 2007 y Díaz-Pulido et al., 2012).

El nivel de estudios también es relevante a la hora de explicar la satisfacción con los servicios públicos en España, pues la probabilidad de encontrarse altamente satisfechos de quienes han cursado estudios de formación profesional o cuentan con una titulación universitaria media disminuye un 17 y un $24 \%$, respectivamente, en comparación con quienes tienen estudios universitarios superiores. Una de las posibles causas podría ser que el nivel de estudios está alta y directamente relacionado con la situación económica, por lo que los ciudadanos mejor formados disfrutan de una posición más elevada y desahogada. En consecuencia, necesitan en 
menor medida la cobertura de estos servicios públicos. Esta relación entre el nivel de estudios y los altos niveles de satisfacción se aprecia mejor en los resultados obtenidos a partir de las regresiones individuales para cada una de las modalidades consideradas de servicio público. Así, son precisamente quienes tienen estudios universitarios superiores quienes acceden a las prestaciones por desempleo y a las pensiones en mejores condiciones, mientras que, sin embargo, son el colectivo con menor probabilidad de declararse bastante o muy satisfechos con la educación tal vez debido a sus expectativas más elevadas o al mayor nivel crítico respecto a este servicio público.

\section{Discusión y conclusiones}

Las Encuestas sobre "Calidad de los Servicios Públicos" muestran un ligero aumento de la satisfacción de la ciudadanía con el funcionamiento de los servicios públicos en España en el periodo 2009-2011, coincidiendo con un empeoramiento de la coyuntura económica. Atendiendo a la desagregación por servicios, nuestro objetivo ha sido analizar qué ocurre con la satisfacción con la educación, la sanidad, las prestaciones por desempleo y las pensiones, puesto que son los cuatro servicios que suponen un mayor porcentaje del gasto público y que se identifican con el Estado de Bienestar de manera más directa.

En el caso de la primera hipótesis, que relacionaba la satisfacción con el funcionamiento de los servicios públicos con la situación laboral de las personas, la evidencia empírica encuentra una relación muy débil y además sólo en el caso de algunos servicios públicos. La segunda hipótesis vinculaba la satisfacción con el funcionamiento de los servicios públicos con la orientación ideológica de los ciudadanos. En este caso, los datos sí respaldan esta hipótesis, de forma que las personas que se declaran "de izquierdas" tienen una mayor probabilidad de expresar un mayor grado de satisfacción con los servicios públicos.

También se han validado las hipótesis tercera y cuarta, dado que los resultados muestran la relevancia de las comunidades autónomas, especialmente en el caso de los servicios transferidos (educación y sanidad) y una mayor satisfacción de los inmigrantes con los servicios públicos.

Finalmente, la última de las hipótesis planteadas establecía una relación directa entre satisfacción con el funcionamiento de los servicios públicos y el empeoramiento del ciclo económico. Los datos apoyan esta hipótesis, tanto en términos de mayor satisfacción en 2011 que en los años anteriores como de relación directa con la tasa de paro, de forma que una mayor tasa de desempleo afecta positivamente a la valoración de los servicios públicos. Sin embargo, el periodo de tiempo analizado es corto, por lo que sería necesario disponer de datos para un periodo más amplio para confirmar esta hipótesis.

El análisis realizado ha puesto de manifiesto que las variables que tienen un mayor impacto sobre la satisfacción de la ciudadanía con el funcionamiento de los servicios públicos son la ideología auto-declarada, la nacionalidad y el lugar de 
residencia. Nuestros resultados nos permiten concluir que, además de la calidad del servicio, también es muy probable que las expectativas de las personas influyan sobre su satisfacción con los servicios recibidos. Así, las dificultades en los países de origen de quienes emigran posiblemente les hace valorar en mayor medida los servicios públicos existentes en España y las personas "de izquierdas" tienen en su mayoría un mayor compromiso con el Estado de Bienestar que quienes se declaran "de derechas". Estos resultados coinciden en gran medida con los obtenidos por Díaz-Pulido et al. (2012) y, además, mantienen la consistencia a lo largo del trienio analizado. Posteriores análisis que incluyeran las expectativas de los individuos podrían ayudar a entender la opinión de la ciudadanía sobre los servicios públicos. Asimismo, si se pudiera disponer de datos longitudinales, se podría analizar la evolución en el tiempo de dicha opinión. 


\section{Bibliografía}

Agencia Estatal de Evaluación de las Políticas Públicas y la Calidad de los Servicios (2009): La percepción Social de los Servicios Públicos en España (19852008). Ministerio de la Presidencia. Madrid.

Agencia Estatal de Evaluación de las Políticas Públicas y la Calidad de los Servicios (2010): Agenda Pública y satisfacción con los servicios públicos en el Estado Autonómico. Ministerio de la Presidencia. Madrid.

Blekesaune, Morten y Jill Quadagno (2003): "Public Attitudes toward Welfare State Policies: A Comparative Analysis of 24 Nations." European Sociological Review, Vol.19, pp. 415-27. doi: 10.1093/esr/19.5.415

Calzada, Inés y Eloisa del Pino (2008): "Perceived efficacy and citizens' attitudes toward welfare State Reform". International Review of Administrative Science, Vol. 74(4), pp. 31-54. doi: 10.1177/0020852308098468

Calzada, Inés (2007): “¿Qué Estado del Bienestar queremos? Las opiniones de los ciudadanos sobre cómo son y cómo deberían ser nuestras políticas sociales". Zerbitzuan, Revista de Servicios Sociales del Gobierno Vasco, diciembre.

Carrillo, Ernesto y Manuel Tamayo (2008). "El estudio de la opinión pública sobre la administración y las políticas públicas". Gestión y Política Pública, Vol. XVII (1), pp. 193-215.

CIS (varios años): Calidad de los Servicios Públicos. (Estudios 2.908, 2.840, 2.813, 2.762, 2.706, 2.655). Madrid.

Díaz-Pulido, José M., Eloisa del Pino y Pau Palop (2012): "Los determinantes de la satisfacción con las políticas de bienestar del Estado autonómico". Revista Española de Investigaciones Sociológicas, Vol. 139, pp. 45-83. doi: $10.5477 /$ cis/reis. 139.45

Fernández, Juan J., \& Jaime-Castillo, Antonio M. (2013). "Positive or Negative Policy Feedbacks? Explaining Popular Attitudes Towards Pragmatic Pension Policy Reforms". European Sociological Review, 29(4), pp. 803-815. doi: 10.1093/esr/jes059

Fraile, Marta \& Ferrer, Mariona (2005). "Explaining the determinants of public support for cuts in unemployment benefits spending across OECD countries". International Sociology, 20(4), pp. 459-481. doi: 10.1177/0268580905058327

Fundación BBVA (2013): Values and Worldviews: Valores políticos-económicos y la crisis económica.

González, Benjamín y Enrique Carreras (2011). "Los límites de la lealtad ideológica en la evaluación ciudadana del "performance" administrativo local". Papers. Revista de Sociología, Vol. 97(4), pp. 719-749.

Jordan, Jason (2010)." Institutional feedback and support for the welfare state: The case of national health care". Comparative Political Studies, 43(7), pp. 862-885. doi: 10.1177/0010414010361342

Pino, Eloisa del (2005a): “ ¿Se han modificado las preferencias de los ciudadanos sobre las políticas de bienestar en España (1985-2005)?" Unidad de Políticas Comparadas (CSIC). Documento de Trabajo 05-03. 
Pino, Eloisa del (2005b): "Attitudes, performance and institutions: Spanish citizens and public administrations". Public Performance and Management Review, Vol. 28(4), pp. 512-531.

Pino, Eloisa del (2007): "Las actitudes de los españoles hacia la reforma del Estado de Bienestar". Política y Sociedad, Vol. 44(2), pp. 185-208.

Pino, Eloisa del y Juan A. Ramos (2009): "Las reformas de las políticas de bienestar en España: una visión de conjunto", en Moreno, L. (ed), Reformas de las políticas de bienestar en España. Madrid: Siglo XXI, pp. 337-362.

Pino, Eloisa del, Juan A. Ramos y José M. Díaz-Pulido (2012): "Retrenchment in the Spanish welfare state" Workin Paper 136/2012. Departamento de Ciencia Política y Relaciones Internacionales Universidad Autónoma de Madrid.

Pino, Eloisa del, y Díaz-Pulido, José M. (2011): "Lecciones aprendidas desde la experiencia española de análisis de la percepción ciudadana de los servicios públicos”, Reforma y Democracia. Revista del CLAD, Núm. 49, pp. 157-184.

Roch, Christine H. y Theodore H. Poister (2006): "Citizens, Accountability, and Service Satisfaction: The Influence of Expectations" Urban Affairs Review Vol. 41, pp. 292-308. doi: 10.1177/1078087405281124

Taylor-Gooby, Peter (2001): "Sustaining state welfare in hard times: who will foot the bill?". Journal of European Social Policy, Vol. 11(2), pp.133-147. doi: 10.1177/095892870101100203

Vigoda-Gadot, Eran, Aviv Shoham y Dana R. Vashdi (2010): "Bridging bureaucracy and democracy in Europe: A comparative study of perceived managerial excellence, satisfaction with public services, and trust in governance". European Union Politics, Vol. 11(2), pp. 289-30. doi: 10.1177/1465116510363657 
Tabla A.I. Estadísticos descriptivos de las variables utilizadas

\begin{tabular}{|c|c|c|c|c|c|c|c|c|c|}
\hline & & 2009 & & & 2010 & & & 2011 & \\
\hline & $\mathrm{n}$ & media & D.E. & $\mathrm{n}$ & media & D.E. & $\mathrm{n}$ & media & D.E. \\
\hline Sexo & 7924 & 0,490 & 0,500 & 2489 & 0,494 & 0,500 & 2461 & 0,491 & 0,500 \\
\hline Edad & 7924 & 46,6 & 17,8 & 2489 & 46,7 & 17,8 & 2461 & 47,1 & 17,8 \\
\hline $\begin{array}{l}\text { Nacionalidad (extran- } \\
\text { jera) }\end{array}$ & 7917 & 0.072 & 0,259 & 2484 & 0.076 & 0,265 & 2450 & 0.076 & 0,266 \\
\hline Situación profesional: & & & & & & & & & \\
\hline Trabaja & 7913 & 0,446 & 0,497 & 2484 & 0,450 & 0,498 & 2456 & 0,447 & 0,497 \\
\hline Jubilado o pensionista & 7913 & 0,184 & 0,388 & 2484 & 0,188 & 0,391 & 2456 & 0,184 & 0,387 \\
\hline $\begin{array}{l}\text { Pensionista (previamen- } \\
\text { te no ha trabajado) }\end{array}$ & 7913 & 0,037 & 0,190 & 2484 & 0,027 & 0,161 & 2456 & 0.035 & 0.185 \\
\hline Parado con experiencia & 7913 & 0,177 & 0,381 & 2484 & 0,190 & 0,392 & 2456 & 0,190 & 0,392 \\
\hline Parado sin experiencia & 7913 & 0,008 & 0,090 & 2484 & 0,006 & 0,080 & 2456 & 0,011 & 0,102 \\
\hline Estudiante & 7913 & 0,042 & 0,200 & 2484 & 0,034 & 0,182 & 2456 & 0,043 & 0,202 \\
\hline $\begin{array}{l}\text { Trabajo doméstico no } \\
\text { remunerado }\end{array}$ & 7913 & 0,100 & 0,300 & 2484 & 0,100 & 0,300 & 2456 & 0,085 & 0,278 \\
\hline Otra situación & 7913 & 0,005 & 0,073 & 2484 & 0,005 & 0,069 & 2456 & 0,007 & 0,080 \\
\hline Estudios: & & & & & & & & & \\
\hline Sin estudios & 7900 & 0,082 & 0,275 & 2482 & 0,076 & 0,265 & 2460 & 0,073 & 0,260 \\
\hline Primaria & 7900 & 0,439 & 0,496 & 2482 & 0,452 & 0,498 & 2460 & 0,454 & 0,498 \\
\hline Secundaria & 7900 & 0,138 & 0,345 & 2482 & 0,130 & 0,336 & 2460 & 0,129 & 0,335 \\
\hline Formación Profesional & 7900 & 0,149 & 0,356 & 2482 & 0,159 & 0,366 & 2460 & 0,143 & 0,351 \\
\hline Medios universitarios & 7900 & 0,081 & 0,272 & 2482 & 0,080 & 0,271 & 2460 & 0,091 & 0,287 \\
\hline Superiores & 7900 & 0,110 & 0,313 & 2482 & 0,103 & 0,304 & 2460 & 0,109 & 0,312 \\
\hline Estatus: & & & & & & & & & \\
\hline Clase alta/media-alta & 7757 & 0,171 & 0,377 & 2419 & 0,162 & 0,369 & 2415 & 0,177 & 0,382 \\
\hline Nuevas clases medias & 7757 & 0,192 & 0,394 & 2419 & 0,208 & 0,406 & 2415 & 0,197 & 0,398 \\
\hline Viejas clases medias & 7757 & 0,163 & 0,370 & 2419 & 0,162 & 0,369 & 2415 & 0,155 & 0,362 \\
\hline Obreros cualificados & 7757 & 0,326 & 0,469 & 2419 & 0,319 & 0,466 & 2415 & 0,333 & 0,471 \\
\hline Obreros no cualificados & 7757 & 0,147 & 0,355 & 2419 & 0,148 & 0,355 & 2415 & 0,139 & 0,346 \\
\hline $\begin{array}{l}\text { Condición socioeco- } \\
\text { nómica: }\end{array}$ & & & & & & & & & \\
\hline $\begin{array}{l}\text { Directores y profesiona- } \\
\text { les }\end{array}$ & 7924 & 0,038 & 0,191 & 2489 & 0,043 & 0,202 & 2461 & 0,038 & 0,192 \\
\hline $\begin{array}{l}\text { Técnicos y cuadros } \\
\text { medios }\end{array}$ & 7924 & 0,101 & 0,302 & 2489 & 0,098 & 0,297 & 2461 & 0,092 & 0,289 \\
\hline Pequeños empresarios & 7924 & 0,034 & 0,181 & 2489 & 0,037 & 0,188 & 2461 & 0,040 & 0,196 \\
\hline Agricultores & 7924 & 0,009 & 0,093 & 2489 & 0,007 & 0,082 & 2461 & 0,006 & 0,075 \\
\hline Empleados de oficinas & & & & & & & & & \\
\hline y servicio & 7924 & 0,070 & 0,255 & 2489 & 0,072 & 0,258 & 2461 & 0,073 & 0,260 \\
\hline Obreros cualificados & 7924 & 0,082 & 0,275 & 2489 & 0,074 & 0,262 & 2461 & 0,084 & 0,278 \\
\hline Obreros no cualificados & 7924 & 0,102 & 0,303 & 2489 & 0,109 & 0,312 & 2461 & 0,105 & 0,307 \\
\hline Jubilados y pensionistas & 7924 & 0,222 & 0,415 & 2489 & 0,214 & 0,410 & 2461 & 0,219 & 0,413 \\
\hline Parados & 7924 & 0,185 & 0,388 & 2489 & 0,196 & 0,397 & 2461 & 0,200 & 0,400 \\
\hline Estudiantes & 7924 & 0,042 & 0,200 & 2489 & 0,034 & 0,182 & 2461 & 0,043 & 0,202 \\
\hline Trabajo doméstico no & & & & & & & & & \\
\hline $\begin{array}{l}\text { remunerado } \\
\text { Noclasificables }\end{array}$ & 7924 & 0,100 & 0,300 & 2489 & 0,100 & 0,300 & 2461 & 0,085 & 0,278 \\
\hline $\begin{array}{l}\text { No clasificables } \\
\text { Ocupación: }\end{array}$ & 7924 & 0,016 & 0,125 & 2489 & 0,016 & 0,127 & 2461 & 0,016 & 0,126 \\
\hline $\begin{array}{l}\text { Dirección de empresas } \\
\text { y administración }\end{array}$ & 7846 & 0,072 & 0,258 & 2443 & 0.059 & 0236 & 2435 & 0.069 & 0.253 \\
\hline Técnicos y prof. & & & & & & & & & \\
\hline $\begin{array}{l}\text { clentificos e intelectua- } \\
\text { les }\end{array}$ & 7846 & 0,120 & 0,325 & 2443 & 0,107 & 0,309 & 2435 & 0,120 & 0,325 \\
\hline $\begin{array}{l}\text { Técnicos y profesiona- } \\
\text { les de apoyo }\end{array}$ & 7846 & 0,113 & 0,317 & 2443 & 0,130 & 0,336 & 2435 & 0,117 & 0,322 \\
\hline
\end{tabular}




\begin{tabular}{|c|c|c|c|c|c|c|c|c|c|}
\hline & \multicolumn{3}{|c|}{2009} & \multicolumn{3}{|c|}{2010} & \multicolumn{3}{|c|}{2011} \\
\hline & $\mathrm{n}$ & media & D.E. & $\mathrm{n}$ & media & D.E. & $\mathrm{n}$ & media & D.E. \\
\hline administrativo & 7846 & 0,042 & 0,201 & 2443 & 0,040 & 0,195 & 2435 & 0,034 & 0,180 \\
\hline $\begin{array}{l}\text { servicios de restaura- } \\
\text { ción }\end{array}$ & 7846 & 0,138 & 0,345 & 2443 & 0,153 & 0,360 & 2435 & 0,165 & 0,371 \\
\hline $\begin{array}{l}\text { Trabajadores cualifica- } \\
\text { dos de agricultura }\end{array}$ & 7846 & 0,063 & 0,243 & 2443 & 0,062 & 0,241 & 2435 & 0,063 & 0,243 \\
\hline $\begin{array}{l}\text { Artesanos y trabajado- } \\
\text { res cualificados }\end{array}$ & 7846 & 0,188 & 0,391 & 2443 & 0,185 & 0,388 & 2435 & 0,175 & 0,380 \\
\hline $\begin{array}{l}\text { Operadores de maqui- } \\
\text { naria y montadores }\end{array}$ & 7846 & 0,120 & 0,325 & 2443 & 0,120 & 0,325 & 2435 & 0,128 & 0,334 \\
\hline $\begin{array}{l}\text { Trabajadores no } \\
\text { cualificados }\end{array}$ & 7846 & 0,138 & 0,345 & 2443 & 0,138 & 0,345 & 2435 & 0,126 & 0,332 \\
\hline $\begin{array}{l}\text { Fuerzas armadas } \\
\text { Orientación política: }\end{array}$ & 7846 & 0,007 & 0,081 & 2443 & 0,006 & 0,075 & 2435 & 0,004 & 0,064 \\
\hline Izquierda & 7924 & 0,154 & 0,361 & 2489 & 0,165 & 0,371 & 2461 & 0,166 & 0,372 \\
\hline Centro-izquierda & 7924 & 0,127 & 0,333 & 2489 & 0,121 & 0,326 & 2461 & 0,124 & 0,330 \\
\hline Centro & 7924 & 0,227 & 0,419 & 2489 & 0,199 & 0,400 & 2461 & 0,199 & 0,399 \\
\hline Centro-derecha & 7924 & 0,086 & 0,280 & 2489 & 0,086 & 0,280 & 2461 & 0,086 & 0,281 \\
\hline Derecha & 7924 & 0,122 & 0,328 & 2489 & 0,111 & 0,315 & 2461 & 0,138 & 0,345 \\
\hline $\mathrm{Ns} / \mathrm{nc}$ & 7924 & 0,284 & 0,451 & 2489 & 0,317 & 0,466 & 2461 & 0,286 & 0,452 \\
\hline $\begin{array}{l}\text { Votó en las últimas } \\
\text { elecciones }\end{array}$ & 7891 & 0,564 & 0,496 & 2483 & 0,537 & 0,499 & 2456 & 0,556 & 0,497 \\
\hline
\end{tabular}

Fuente: Elaboración propia a partir de las encuestas sobre "Calidad de los Servicios Públicos" 2009-2011. CIS. 
Tabla A.I.I: Determinantes de la probabilidad de que la ciudadanía esté muy o bastante satisfecha con el funcionamiento de los servicios públicos (regresiones logísticas)

\begin{tabular}{|c|c|c|c|c|c|c|c|c|c|c|c|c|c|c|c|}
\hline & \multicolumn{3}{|c|}{$\begin{array}{l}\text { Servicios públicos } \\
\quad(\mathrm{n}=12.100)\end{array}$} & \multicolumn{3}{|c|}{$\begin{array}{l}\text { Sanidad } \\
(12.012)\end{array}$} & \multicolumn{3}{|c|}{$\begin{array}{l}\text { Educación } \\
(n=10.798)\end{array}$} & \multicolumn{3}{|c|}{$\begin{array}{c}\text { Prestaciones } \\
\text { desempleo } \\
(\mathbf{n}=9.618)\end{array}$} & \multicolumn{3}{|c|}{$\begin{array}{l}\text { Pensiones } \\
(n=9.645)\end{array}$} \\
\hline & R.P.R. & & D.E. & R.P.R. & & D.E. & R.P.R. & & D.E. & R.P.R. & & D.E. & R.P.R. & & D.E. \\
\hline \multicolumn{16}{|c|}{ Sexo (C.R.: mujer) } \\
\hline Hombre & 1,082 & $*$ & 0,045 & 1,298 & $* * *$ & 0,055 & 0,887 & $* * *$ & 0,039 & 1,062 & & 0,049 & 1,17 & $* * *$ & 0,054 \\
\hline Edad & 0,959 & $* * *$ & 0,006 & 0,963 & $* * *$ & 0,007 & 0,983 & $* *$ & 0,007 & 0,99 & & 0,007 & 0,982 & $* * *$ & 0,007 \\
\hline Edad $^{2}$ & 1,001 & $* * *$ & 0 & 1,001 & $* * *$ & 0 & 1 & $* *$ & 0 & 1 & $* *$ & 0 & 1 & $* * *$ & 0 \\
\hline \multicolumn{16}{|c|}{ Español (C.R.: no) } \\
\hline Sí & 0,264 & $* * *$ & 0,332 & 0,342 & $* * *$ & 0,261 & 0,365 & $* * *$ & 0,262 & 0,38 & $* * *$ & 0,231 & 0,346 & $* * *$ & 0,327 \\
\hline \multicolumn{16}{|c|}{ Comunidad autónoma (C.R.: Madrid) } \\
\hline Andalucía & 0,686 & & 0,206 & 0,568 & * & 0,175 & 2,157 & $* *$ & 0,702 & 0,939 & & 0,318 & 0,552 & $*$ & 0,182 \\
\hline Aragón & 1,483 & $* * *$ & 0,206 & 2,537 & $* * *$ & 0,365 & 2,771 & $* * *$ & 0,419 & 2,102 & $* * *$ & 0,325 & 1,347 & $*$ & 0,207 \\
\hline Asturias & 3,223 & $* * *$ & 0,479 & 4,87 & $* * *$ & 0,806 & 6,023 & $* * *$ & 1,064 & 3,883 & $* * *$ & 0,61 & 1,965 & $* * *$ & 0,298 \\
\hline Baleares & 0,877 & & 0,146 & 2,035 & $* * *$ & 0,356 & 1,903 & $* * *$ & 0,338 & 1,429 & $*$ & 0,265 & 0,707 & $*$ & 0,134 \\
\hline Canarias & 0,358 & $* * *$ & 0,112 & 0,236 & $* * *$ & 0,075 & 1,503 & & 0,505 & 0,707 & & 0,248 & 0,323 & $* * *$ & 0,111 \\
\hline Cantabria & 1,674 & $* * *$ & 0,299 & 1,531 & $* *$ & 0,274 & 1,659 & $* * *$ & 0,32 & 0,94 & & 0,209 & 1,037 & & 0,217 \\
\hline $\begin{array}{l}\text { Castilla la } \\
\text { Mancha }\end{array}$ & 0,81 & & 0,116 & 1,495 & $* * *$ & 0,219 & 1,73 & $* * *$ & 0,265 & 1,276 & & 0,207 & 1,001 & & 0,161 \\
\hline $\begin{array}{l}\text { Castilla y } \\
\text { León }\end{array}$ & 1,598 & $* * *$ & 0,167 & 1,791 & $* * *$ & 0,19 & 1,87 & $* * *$ & 0,209 & 1,662 & $* * *$ & 0,197 & 1,38 & $* * *$ & 0,16 \\
\hline Cataluña & 0,896 & & 0,082 & 1,162 & & 0,108 & 1,185 & $*$ & 0,116 & 1,187 & $*$ & 0,123 & 0,637 & $* * *$ & 0,065 \\
\hline $\begin{array}{l}\text { Comunidad } \\
\text { Valenciana }\end{array}$ & 0,689 & $*$ & 0,138 & 1,102 & & 0,224 & 1,632 & $* *$ & 0,348 & 1,043 & & 0,232 & 0,676 & $*$ & 0,149 \\
\hline Extremadura & 0,657 & $*$ & 0,153 & 0,68 & & 0,161 & 2,049 & $* * *$ & 0,512 & 1,145 & & 0,295 & 0,431 & $* * *$ & 0,111 \\
\hline Galicia & 1,646 & $* * *$ & 0,164 & 1,541 & $* * *$ & 0,155 & 2,219 & $* * *$ & 0,24 & 1,305 & $* *$ & 0,147 & 0,979 & & 0,107 \\
\hline Murcia & 0,833 & & 0,19 & 0,979 & & 0,226 & 2,387 & $* * *$ & 0,586 & 1,13 & & 0,289 & 0,575 & $* *$ & 0,148 \\
\hline Navarra & 3,097 & $* * *$ & 0,591 & 4,395 & $* * *$ & 0,926 & 3,781 & $* * *$ & 0,808 & 2,039 & $* * *$ & 0,439 & 1,553 & & 0,33 \\
\hline País Vasco & 1,544 & $* * *$ & 0,173 & 3,468 & $* * *$ & 0,425 & 3,367 & $* * *$ & 0,427 & 1,219 & & 0,155 & 0,976 & & 0,123 \\
\hline La Rioja & 2,577 & $* * *$ & 0,658 & 2,153 & $* * *$ & 0,546 & 4,582 & $* * *$ & 1,381 & 1,891 & $* *$ & 0,479 & 2,203 & $* * *$ & 0,583 \\
\hline \multicolumn{16}{|c|}{ Tamaño del municipio (C.R.: 10.001-50.000) } \\
\hline$\leq 2.000$ & 1,031 & & 0,09 & 1,107 & & 0,099 & 1,088 & & 0,103 & 1,178 & * & 0,114 & 1,234 & $* *$ & 0,115 \\
\hline $2.001-10.000$ & 0,973 & & 0,059 & 1,196 & $* * *$ & 0,075 & 1,036 & & 0,068 & 1,009 & & 0,068 & 0,981 & & 0,065 \\
\hline $\begin{array}{l}50.001- \\
100.000 \\
\end{array}$ & 1,036 & & 0,069 & 1,169 & $* *$ & 0,079 & 1,056 & & 0,076 & 0,961 & & 0,07 & 1,049 & & 0,078 \\
\hline $\begin{array}{l}100.001- \\
400.000\end{array}$ & 1,051 & & 0,058 & 1,211 & $* * *$ & 0,069 & 0,985 & & 0,058 & 0,899 & $*$ & 0,055 & 0,888 & $*$ & 0,054 \\
\hline $\begin{array}{l}400.001- \\
1.000 .000\end{array}$ & 0,851 & $*$ & 0,074 & 0,914 & & 0,081 & 0,64 & $* * *$ & 0,059 & 0,869 & & 0,087 & 0,795 & $* *$ & 0,079 \\
\hline$>1.000 .000$ & 1,283 & $* * *$ & 0,107 & 1,701 & $* * *$ & 0,146 & 1,094 & & 0,097 & 0,973 & & 0,092 & 0,879 & & 0,082 \\
\hline
\end{tabular}




\begin{tabular}{|c|c|c|c|c|c|c|c|c|c|c|c|c|c|c|c|}
\hline & \multicolumn{3}{|c|}{$\begin{array}{l}\text { Servicios públicos } \\
\quad(n=12.100)\end{array}$} & \multicolumn{3}{|c|}{$\begin{array}{l}\text { Sanidad } \\
(\mathbf{1 2 . 0 1 2})\end{array}$} & \multicolumn{3}{|c|}{$\begin{array}{l}\text { Educación } \\
(\mathrm{n}=10.798)\end{array}$} & \multicolumn{3}{|c|}{$\begin{array}{c}\text { Prestaciones } \\
\text { desempleo } \\
(\mathbf{n}=9.618)\end{array}$} & \multicolumn{3}{|c|}{$\begin{array}{l}\text { Pensiones } \\
(\mathrm{n}=9.645)\end{array}$} \\
\hline & R.P.R. & \multicolumn{2}{|c|}{ D.E. } & R.P.R. & \multicolumn{2}{|c|}{ D.E. } & R.P.R. & & D.E. & R.P.R. & & D.E. & R.P.R. & & D.E. \\
\hline \multicolumn{16}{|c|}{ Situación laboral (C.R. trabaja) } \\
\hline Pensionista & 1,033 & \multicolumn{2}{|r|}{0,077} & 1,012 & \multicolumn{2}{|r|}{0,078} & \begin{tabular}{l|l}
8 & 0,954
\end{tabular} & \multicolumn{2}{|r|}{0,079} & 1,089 & \multicolumn{2}{|r|}{0,092} & 1,081 & \multicolumn{2}{|r|}{0,083} \\
\hline Desempleado & 0,758 & $* * *$ & 0,04 & 1,008 & \multicolumn{2}{|r|}{0,054} & \begin{tabular}{l|l}
4 & 0,948
\end{tabular} & & 856 & $* * *$ & 0,048 & 3 & \multicolumn{2}{|r|}{0,059} \\
\hline Otros inactivos & 1,138 & $* *$ & 0,075 & 1,155 & $* *$ & 0,077 & \begin{tabular}{l|l}
7 & 0,945
\end{tabular} & & 0,067 & \begin{tabular}{l|l}
7 & 0,948
\end{tabular} & & 0,072 & 1,032 & & 0,077 \\
\hline Estudios (C.R.: u & universita & os su & eriores & & & & & & & & & & & & \\
\hline Sin estudios & 0,829 & & 0,095 & 0,933 & & 0,111 & $1 \quad 1,842$ & $* * *$ & 0,232 & 0,69 & $* * *$ & 0,091 & 0,632 & $* * *$ & 0,079 \\
\hline Primaria & 0,973 & & 0,082 & 0,925 & & 0,08 & 1,706 & $* * *$ & 0,152 & \begin{tabular}{l|l}
2 & 0,793
\end{tabular} & $* *$ & 0,074 & 0,806 & $* *$ & 0,077 \\
\hline Secundaria & 0,925 & & 0,082 & 0,963 & & 0,087 & \begin{tabular}{l|l}
7 & 1,268
\end{tabular} & $* *$ & 0,117 & \begin{tabular}{l|l}
7 & 0,806
\end{tabular} & $* *$ & 0,079 & 0,841 & $*$ & 0,085 \\
\hline $\begin{array}{l}\text { Formación } \\
\text { Profesional }\end{array}$ & 0,854 & $*$ & 0,075 & 0,871 & & 0,078 & \begin{tabular}{l|l}
8 & 1,309
\end{tabular} & $* * *$ & 0,119 & 9 & $* *$ & 0,078 & 0,802 & $* *$ & 0,079 \\
\hline $\begin{array}{l}\text { Medios universita } \\
\text { rios }\end{array}$ & 0,808 & $* *$ & 0,07 & 0,836 & $* *$ & 0,075 & \begin{tabular}{l|l}
5 & 0,978
\end{tabular} & & 0,088 & \begin{tabular}{l|l}
3 & 0,937
\end{tabular} & & 0,091 & 1,068 & & 0,105 \\
\hline Status (C.R.: obr & reros no cl & AIIIC & dos) & & & & & & & & & & & & \\
\hline $\begin{array}{l}\text { Clase alta / media } \\
\text { alta }\end{array}$ & 1,132 & & 0,209 & 0,894 & & 0,169 & \begin{tabular}{l|l}
9 & 0,929
\end{tabular} & & 0,183 & \begin{tabular}{l|l}
3 & 1,228
\end{tabular} & & 0,255 & 0,907 & & 0,191 \\
\hline $\begin{array}{l}\text { Nuevas clases } \\
\text { medias }\end{array}$ & 1,173 & & 0,192 & 0,825 & & 0,137 & \begin{tabular}{l|l}
7 & 0,938
\end{tabular} & & 0,163 & \begin{tabular}{l|l}
3 & 1,271
\end{tabular} & & 0,23 & 0,974 & & 0,182 \\
\hline $\begin{array}{l}\text { Viejas clases } \\
\text { medias }\end{array}$ & 0,986 & & 0,175 & 0,989 & & 0,179 & \begin{tabular}{l|l}
9 & 0,981
\end{tabular} & & 0,186 & \begin{tabular}{l|l}
6 & 1,207
\end{tabular} & & 0,239 & 1,012 & & 0,204 \\
\hline $\begin{array}{l}\text { Obreros cualifica- } \\
\text { dos }\end{array}$ & 1,113 & & 0,183 & 0,922 & & 0,154 & \begin{tabular}{l|l}
4 & 1,041
\end{tabular} & & 0,182 & \begin{tabular}{l|l}
2 & 1,257
\end{tabular} & & 0,228 & 0,991 & & 0,186 \\
\hline
\end{tabular}

Ocupación (C.R.: técnicos y profesionales de apoyo)

\begin{tabular}{|l|l|l|l|l|l|l|l|l|l|l|l|l|l|l|l|}
\hline $\begin{array}{l}\text { Dirección } \\
\text { empresas y admón. }\end{array}$ & 0,873 & & 0,111 & 0,848 & & 0,11 & 0,989 & & 0,134 & 0,886 & & 0,127 & 0,79 & $*$ & 0,111 \\
\hline $\begin{array}{l}\text { Técnicos y profes. } \\
\text { cient. e intelect. }\end{array}$ & 1,083 & & 0,118 & 1,111 & & 0,124 & 1,181 & & 0,135 & 1,037 & & 0,128 & 1,025 & 0,127 \\
\hline $\begin{array}{l}\text { Empleados de tipo } \\
\text { administrativo }\end{array}$ & 0,902 & & 0,101 & 0,91 & & 0,104 & 0,995 & & 0,116 & 0,987 & & 0,124 & 1,092 & 0,134 \\
\hline $\begin{array}{l}\text { Trabajadores serv. } \\
\text { restauración }\end{array}$ & 0,844 & $*$ & 0,075 & 0,929 & & 0,083 & 0,935 & & 0,087 & 0,984 & & 0,097 & 0,92 & & 0,09 \\
\hline $\begin{array}{l}\text { Trabajadores } \\
\text { cualif. agricultura }\end{array}$ & 1,224 & & 0,177 & 1,051 & & 0,156 & 1,491 & $* *$ & 0,237 & 1,121 & & 0,183 & 0,924 & & 0,146 \\
\hline $\begin{array}{l}\text { Artesanos y trabaj. } \\
\text { cualificados }\end{array}$ & 0,938 & & 0,11 & 0,942 & & 0,112 & 1,128 & & 0,14 & 1,025 & & 0,133 & 0,827 & & 0,108 \\
\hline $\begin{array}{l}\text { Operadores de } \\
\text { maquin. y montad. }\end{array}$ & 0,883 & & 0,107 & 0,862 & & 0,106 & 1,094 & & 0,141 & 1,042 & & 0,14 & 0,917 & & 0,124 \\
\hline $\begin{array}{l}\text { Trabajadores no } \\
\text { cualificados }\end{array}$ & 1,138 & & 0,207 & 0,866 & & 0,16 & 1,164 & & 0,225 & 1,182 & & 0,239 & 0,87 & & 0,18 \\
\hline Fuerzas armadas & 1,01 & & 0,277 & 1,097 & & 0,312 & 0,727 & & 0,213 & 0,921 & & 0,29 & 0,917 & 0,269 \\
\hline
\end{tabular}

Orientación política (C.R.: izquierda)

\begin{tabular}{|l|c|c|c|c|c|c|c|c|c|c|c|c|c|c|c|}
\hline Centro-izquierda & 1,177 & $* *$ & 0,082 & 1,02 & & 0,073 & 1,064 & & 0,079 & 1,159 & $* *$ & 0,087 & 1,293 & $* * *$ & 0,098 \\
\hline Centro & 1,115 & $*$ & 0,07 & 1,042 & & 0,067 & 0,932 & & 0,062 & 1,017 & & 0,069 & 0,971 & & 0,067 \\
\hline Centro-derecha & 0,863 & $*$ & 0,069 & 0,894 & & 0,073 & 0,687 & $* * *$ & 0,058 & 0,784 & $* * *$ & 0,07 & 0,787 & $* * *$ & 0,069 \\
\hline Derecha & 0,855 & $*$ & 0,062 & 0,851 & $* *$ & 0,063 & 0,755 & $* * *$ & 0,058 & 0,701 & $* * *$ & 0,056 & 0,728 & $* * *$ & 0,056 \\
\hline NS/NC & 0,886 & $*$ & 0,056 & 0,823 & $* * *$ & 0,053 & 0,799 & $* * *$ & 0,054 & 0,891 & & 0,063 & 0,884 & $*$ & 0,062 \\
\hline
\end{tabular}




\begin{tabular}{|c|c|c|c|c|c|c|c|c|c|c|c|c|c|c|c|}
\hline & \multicolumn{3}{|c|}{$\begin{array}{l}\text { Servicios públicos } \\
\quad(n=12.100)\end{array}$} & \multicolumn{3}{|c|}{$\begin{array}{l}\text { Sanidad } \\
(12.012)\end{array}$} & \multicolumn{3}{|c|}{$\begin{array}{l}\text { Educación } \\
(\mathrm{n}=10.798)\end{array}$} & \multicolumn{3}{|c|}{$\begin{array}{c}\text { Prestaciones } \\
\text { desempleo } \\
(\mathbf{n}=\mathbf{9 . 6 1 8})\end{array}$} & \multicolumn{3}{|c|}{$\begin{array}{l}\text { Pensiones } \\
(\mathrm{n}=9.645)\end{array}$} \\
\hline & R.P.R. & & D.E. & R.P.R. & & D.E. & R.P.R. & & D.E. & R.P.R. & & D.E. & R.P.R. & & D.E. \\
\hline \multicolumn{16}{|c|}{ Votó en las últimas elecciones (C.R. no) } \\
\hline Sí & 1,172 & $* * *$ & 0,052 & 1,068 & & 0,048 & 1,032 & & 0,049 & 1,173 & $* * *$ & 0,059 & 1,264 & $* * *$ & 0,062 \\
\hline \multicolumn{16}{|c|}{ Tasa autonómica de desempleo (C.R.: 10-<15\%) } \\
\hline$<10 \%$ & 0,739 & $* * *$ & 0,064 & 0,844 & $*$ & 0,073 & 0,883 & & 0,082 & 0,839 & $*$ & 0,081 & 0,785 & $* * *$ & 0,073 \\
\hline $15-<20 \%$ & 1,554 & $* * *$ & 0,252 & 1,22 & & 0,203 & 1,014 & & 0,175 & 1,044 & & 0,188 & 1,387 & $*$ & 0,249 \\
\hline $20-<25 \%$ & 1,532 & & 0,42 & 2,335 & $* * *$ & 0,659 & 0,746 & & 0,222 & 1,49 & & 0,462 & 1,771 & * & 0,534 \\
\hline \multicolumn{16}{|c|}{ Oleada (C.R.: 2011) } \\
\hline 2009 & 0,627 & $* * *$ & 0,038 & 0,963 & & 0,06 & 0,722 & $* * *$ & 0,047 & 0,863 & $* *$ & 0,058 & 1,352 & $* * *$ & 0,089 \\
\hline 2010 & 0,755 & $* * *$ & 0,048 & 0,751 & $* * *$ & 0,048 & 0,9 & & 0,061 & 1,011 & & 0,071 & 1,112 & & 0,076 \\
\hline
\end{tabular}

C.R.: categoría de referencia.

$(* * *)$ Nivel de significatividad 0,01 .

(**) Nivel de significatividad 0,05.

(*) Nivel de significatividad 0,1.

Fuente: Elaboración propia a partir de las encuestas sobre "Calidad de los Servicios Públicos" 2009-2011. CIS. 